\title{
ANALYSIS ON THE VARIATION OF MEDIAL ROTATION VALUES ACCORDING TO THE POSITION OF THE HUMERAL DIAPHYSIS
}

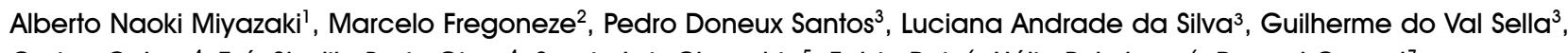
Carina Cohen ${ }^{4}$, Taís Stedile Busin Giora ${ }^{4}$, Sergio Luiz Checchia ${ }^{5}$, Fabio Raia ${ }^{6}$, Hélio Pekelman ${ }^{6}$, Raquel Cymrot ${ }^{7}$

\section{ABSTRACT}

Objective: To analyze the validity of measurements of medial rotation (MR) of the shoulder, using vertebral levels, according to the variation in the position of the humeral diaphysis, and to test the bi-goniometer as a new measuring instrument. Methods: 140 shoulders (70 patients) were prospectively evaluated in cases presenting unilateral shoulder MR limitation. The vertebral level was evaluated by means of a visual scale and was correlated with the angle obtained according to the position of the humeral diaphysis, using the bi-goniometer developed with the Department of Mechanical Engineering of Mackenzie University. Results: The maximum vertebral level reached through MR on the unaffected side ranged from T3 to T12, and on the affected side, from T6 to the trochanter. Repositioning of the affected limb in MR according to the angular values on the normal side showed that $57.13 \%$ of the patients reached lower levels, between the sacrum, gluteus and trochanter. From analysis on the maximum vertebral level attained and the variation between the affected angle $\mathrm{x}$ (frontal plane: abduction and MR of the shoulder) and the unaffected angle $\mathrm{x}$ in MR, we observed that the greater the angle of the diaphyseal axis was, the lower the variation in the vertebral level attained was. From evaluating the linear correlation between the variables of difference in maximum vertebral level reached and variation in the affected angle y (extension and abduction of the shoulder) and the unaffected angle $y$ in MR, we observed that there was no well-established linear relationship between these variables. Conclusion: Measurement of MR using vertebral levels does not correspond to the real values, since it varies according to the positioning of the humeral diaphysis.

Keywords - Shoulder Joint; Range of Motion, Articular; Rotation; Articular, Goniometry

\section{INTRODUCTION}

Range of motion (ROM) is an important parameter in functional evaluations of the shoulder and precision is needed in measuring $\mathrm{it}^{(1)}$. These measurements can be made as specified in the manual of the American Academy of Orthopaedic Surgeons (AAOS), using a visual scale and goniometry ${ }^{(2)}$. Measurements on the medial rotation of the shoulder are particularly difficult to define, because the abdomen impedes the maximum medial rotation. One method that is commonly used is to indirectly measure the medial rotation in terms of the maximum proximal vertebral

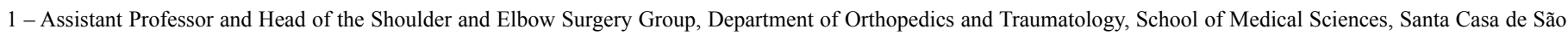
Paulo, São Paulo, Brazil.

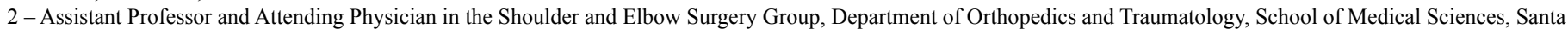
Casa de São Paulo, São Paulo, Brazil.

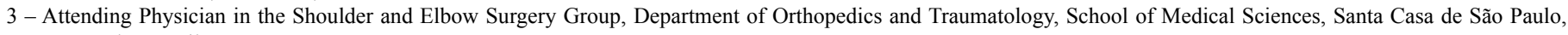
São Paulo, Brazil.

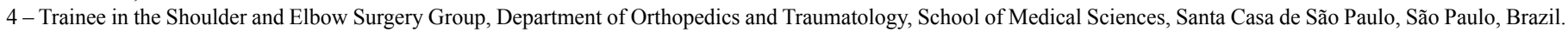

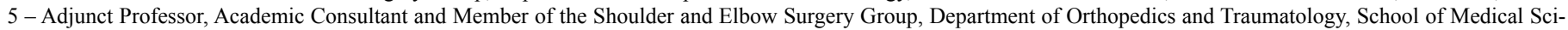
ences, Santa Casa de São Paulo, São Paulo, Brazil.

6 - Assistant Professor in the Mechanical Engineering Course, Mackenzie Presbyterian University, São Paulo, Brazil.

7 - Assistant Professor in the Mechanical Engineering Course, Mackenzie Presbyterian University, São Paulo, Brazil.

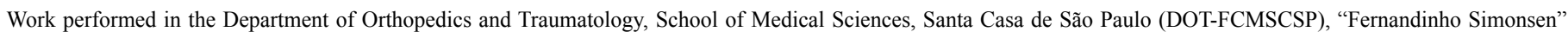

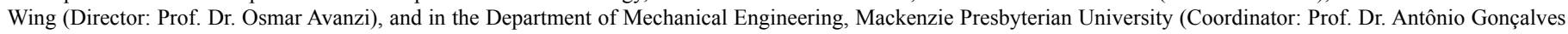
Mello), São Paulo (SP), Brazil.

Correspondence: Rua Dr. Cesário Mota Jr. 112, Vila Buarque, 01221-020 São Paulo, SP. E-mail:lucalu@terra.com.br / ombro@ombro.med.br

Work received for publication: June 2, 2011; accepted for publication: August 19, 2011.

The authors declare that there was no conflict of interest in conducting this work 
level reached by the thumb, in which the hand is actively positioned behind the back and the vertebral level reached by the tip of the extended thumb is recorded ${ }^{(2-5)}$. However, some authors have believed that this measurement underestimates the contribution made by other joints, thus considering that the accuracy of this measurement is not valid ${ }^{(4,6)}$.

In a study comparing the visual estimation method and goniometry for evaluating shoulder ROM, Andrade et $a l^{(1)}$ showed that the greatest reproducibility rate was in measuring the medial rotation using the visual scale method. However, according to Armstrong et $a l^{(7)}$, although visual estimation for evaluating ROM is easy and quickly applied, it presented moderate reproducibility with significant differences in relation to goniometry, which demonstrated high reliability and reproducibility. They therefore considered goniometry to be a better method for evaluating ROM. However, Herrington ${ }^{(8)}$ reported that shoulder measurements made using goniometry could be imprecise because of the difficulty in establishing the axis of movement.

Contrary to what might be believed, medial and lateral rotation do not take place entirely in the shoulder joint ${ }^{(3)}$. Mallon et $a l^{(4)}$ demonstrated that the scapulothoracic joint also contributes towards these movements. The exact amount contributed by these joints varies, with a mean ratio of $2: 1$ at the maximum vertebral levels. Medial rotation along the thorax seems to be done almost entirely by the shoulder joint, which contributes $91 \%$. Taking into account the full medial rotation, the shoulder contributes $84 \%$, but placing the arm behind the back mobilizes the scapulothoracic joint. The movement to position the hand behind the back takes place in three distinct parts: firstly, with the aim in front of the body, the shoulder joint rotates internally; secondly, the arm is positioned behind the body with most of the movement occurring through extension and internal rotation of the scapulothoracic joint; and thirdly, the thumb reaches the vertebral level through elbow flexion. Because this measurement is composed of three movements and does not just consist of medial rotation of the shoulder joint, its value as a medial rotation parameter is questionable. Nonetheless, its functional importance for representing activities of daily living is evident, given that limitation of this movement greatly incapacitates ${ }^{(4,9)}$.

Measurement of medial rotation is part of the standard examination used worldwide ${ }^{(10)}$. The mean vertebral level reached by upper limbs without abnormalities is generally between the thoracic (T) le- vels T6 and T10 ${ }^{(11)}$. Between sacral (S) level S1 and $\mathrm{T} 10$, the movement takes place mainly around elbow flexion $^{(4)}$, and the medial rotation of the shoulder is almost unaltered above $\mathrm{T} 12^{(12)}$.

It is observed that the on the affected side, vertebral levels similar to on the normal side may be reached, at the cost of compensatory inclination of the trunk and elbow flexion. In relation to function, this may appear to be irrelevant; however, for scientific purposes, correct and standardized determination of the vertebral level is fundamental. Seeking to analyze this compensation, we developed a two-dimensional measurement instrument for measuring the angular positioning of the humeral diaphysis in combined medial rotation and extension movements. Through this, we would be able to compare the normal and affected sides, make corrections to the position and compare the vertebral levels reached.

The aim of this study was to analyze the validity of medial rotation measurements on the shoulder using vertebral levels, according to the variation in the position of the humeral diaphysis, and to test the bi-goniometer as a new measurement instrument.

\section{SAMPLE AND METHODS}

Between December 2010 and January 2011, 140 shoulders of 70 patients with unilateral limitation on medial rotation of the shoulder were prospectively evaluated. These patients were being followed up at the outpatient clinic of the Shoulder and Elbow Group, School of Medical Sciences, Santa Casa de São Paulo, "Fernandinho Simonsen" Wing. This research project was approved by the Ethics Committee for Research on Human Beings of this institution. All the patients were aware that they were participating in this study and signed a free and informed consent statement.

The inclusion criterion was that the patients should present unilateral limitation of medial rotation. The following patients were excluded: obese individuals (body mass index, BMI $\geq 30$ ), cases of unconsolidated fracture of an upper limb, cases of limitation of elbow mobility, cases of rheumatoid arthritis, individuals with bilateral shoulder disorders and patients who had undergone shoulder surgery less than four months earlier.

Among the 70 patients evaluated, $41(58.57 \%)$ were female and $29(41.43 \%)$ were male. The mean age was 57 years, ranging from 32 to 82 years. The mean BMI of the patients evaluated was 25.25 (ranging from 17.7 to 29.7 ). The dominant side was affected in 
47 patients $(67.14 \%)$. Forty-five individuals (64.29\%) had pain in the shoulder affected. Forty-nine patients $(70 \%)$ had undergone a surgical procedure, with a mean length of postoperative follow-up of 27 months, ranging from five to 180 months.

The evaluation on the medial rotation of the shoulder was done by means of a visual scale for estimating the vertebral level and this was correlated with the angle obtained according to the position of the diaphysis of the humerus, using a bi-goniometer that had been developed in conjunction with the Department of Mechanical Engineering of Mackenzie University.

\section{Instrument}

The instrument used for the measurements was composed of 18 different pieces, described in the following: piece 1, base, cut from an acrylic board of thickness $15 \mathrm{~mm}$, which served to support the rest of the pieces of the mechanism; piece $2,360^{\circ}$ disc, cut from a polystyrene sheet of thickness $1 \mathrm{~mm}$, which served as a friction-reducing ring, to allow smoother support rotation movements and define the axis for the combination of shoulder extension and adduction (axis of the angle y); piece 3, support, cut from an acrylic board of thickness $15 \mathrm{~mm}$ and then turned and milled to acquire the planned shape, which serves as a support for the half-disc and for the rod; piece 4, $180^{\circ}$ half-disc, made from a cut acrylic protractor, which is responsible for defining the axis of medial rotation and shoulder abduction (axis of the angle $\mathrm{x}$ ); piece 5, rod, cut from a Phenolite tube of length $8 \mathrm{~mm}$, which should be positioned parallel to the humerus, thus making it possible to read the angles; piece 6 , lock, cut from acrylic of thickness $2 \mathrm{~mm}$, which serves to secure the support on the base; piece 7, M3 slotted screw, which fixes the lock to the support; piece 8, bracket, cut from a stainless steel plate, which serves to position the equipment on the patient's body; piece 9, M3 slotted screw, which fixes the support to the base; piece 10, M3 hexagonal nut; piece 11, A3 smooth washer, which helps to fix the support to the base; piece 12, M3 slotted screw, which fixes the rod to the support, thus allowing the rod to rotate; piece 13, upper measurer, prototyped in acrylonitrile butadiene styrene (ABS) plastic, which fixes the spacing between the rod and the lateral edge of the patient's acromion; piece 14, ruler, cut from acrylic of thickness $5 \mathrm{~mm}$ and then milled to obtain parallel clefts, which keeps the upper and lower measurers parallel; piece 15, lower measurer, prototyped in ABS plastic, which should be supported on the lateral epicondyle and then at the tip of the olecranon; piece 16, pin, prototyped in ABS plastic, which fixes the lower measurer to the ruler, thus allowing it to go up and down in parallel with the upper measurer; piece 17, M3 wing nut, which facilitates adjustments to the base; and piece 18 , bubble level, which makes it possible to keep the base horizontally leveled (Figure 1).

The measuring instrument was validated statistically, and its repeatability and reproducibility were analyzed separately. Measurements that did not differ by more than $15^{\circ}$ were considered to be concordant, taking into account the large ROM of the shoulder. Its repeatability was checked through the degree of concordance between the results from successive measurements in the neutral position and in medial rotation, on the same patient, performed using the same method, the same operator and the same conditions of use. Reproducibility was observed in terms of the concordance between the results from measurements in the neutral position and in medial rotation, performed by two operators on the same patient.

\section{Methodology}

All the patients were evaluated by the same two examiners (E1 and E2). The examinations were performed with the patient standing upright, and reference points were marked out on the lateral edges of the acromion, lateral epicondyle and tip of the olecranon of both upper limbs, using colored

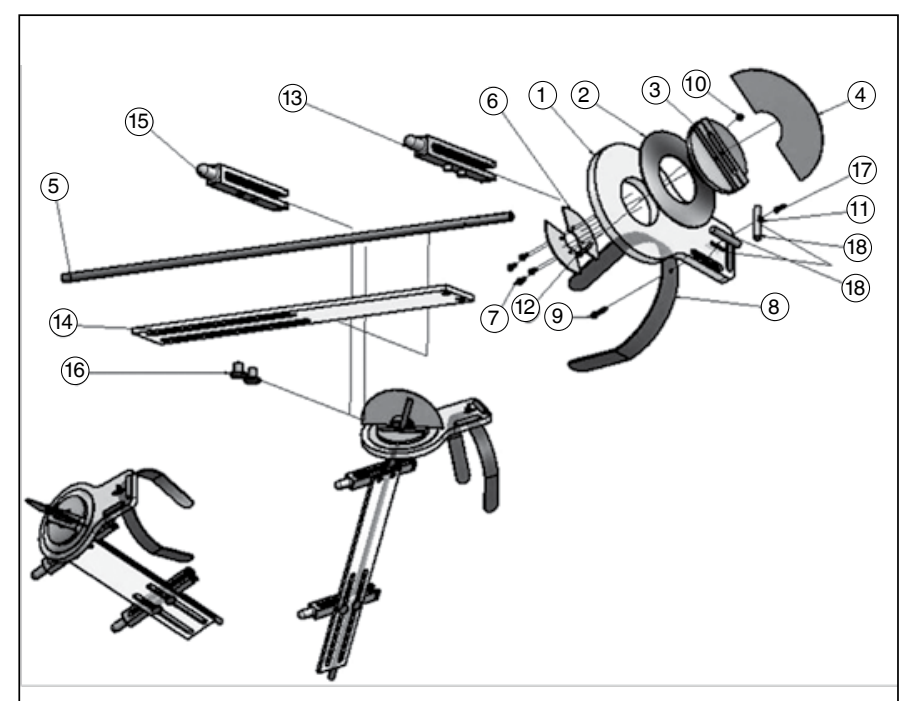

Figure 1 - Illustrative diagram of the bi-goniometer and its components: 1) acrylic base; 2) $360^{\circ}$ polystyrene disc (axis of the angle y); 3) acrylic support; 4) $180^{\circ}$ acrylic protractor (axis of the angle $\mathrm{x}$ ); 5) Phenolite rod; 6) acrylic lock; 7) screws; 8) stainless steel bracket; 9) screws; 10) hexagonal nuts; 11) smooth washers; 12) screws; 13) upper measurer made of ABS plastic; 14) acrylic ruler; 15) lower measurer made of ABS plastic; 16) pins made of ABS plastic; 17) wing nuts; 18) bubble levels. 
adhesive tape. These points were used to determine the alignment of the diaphysis of the humerus.

The bracket of the bi-goniometer was supported on the patient's shoulders, firstly on the normal side. After making measurements in the neutral position and in medial rotation, the same procedure was performed on the affected side. The apparatus was always stabilized by examiner E1, who positioned the midline of the instrument aligned with the lateral edge of the acromion. The levels were carefully observed to certify that the apparatus remained parallel to the ground all the time.

As a starting point, the patient was positioned standing upright with the upper limbs in anatomical position. The angular values obtained from the apparatus were always noted down by examiner E2, using the lateral edge of the acromion and the lateral epicondyle as the parameters, which corresponded to the axis of the humeral diaphysis in this position (Figure 2). Following this, the patient was asked to position the limb in medial rotation on his back, as high as possible, with the thumb outstretched. The apparatus was positioned using the lateral edge of the acromion and the tip of the olecranon as the parameters, which corresponded to the axis of the humeral diaphysis in this position. The angular measurements marked on the apparatus, the degree of elbow flexion and the vertebral level reached by the outstretched thumb were noted down by examiner E2. This sequence was done firstly on the normal side and then on the affected side.

The angular value measured on the unaffected side positioned in medial rotation was used as a parameter for repositioning the affected limb. After alignment was done by examiner E1, a new measurement was made on the maximum vertebral level reached, using the visual scale, and was noted down by examiner E2 (Figure 3).

The angular variation observed between the limb with the limitation on medial rotation, with repositioning of the humeral axis on this side, and the angular values from the normal side was analyzed and compared with the difference in maximum vertebral level reached by the affected member.

For the statistical analysis, the Minitab 16 statistical software was used. In all the hypothesis tests, descriptive levels ( $p$ values) were calculated, taking 95\% confidence intervals to be statistically significant and therefore rejecting hypotheses with $p \leq 0.05$.

To compare means between pairs of variables, Student's t test for paired data was used. Linear correlations between pairs of variables were also calculated, such as the $\Delta \mathrm{x}$ variation, which quantitatively represents the angular variation of the $\mathrm{x}$ axis (angle $\mathrm{x}$ on affected side minus $\mathrm{x}$ on unaffected side, in medial rotation) and the $\Delta y$ variation, quantitatively representing

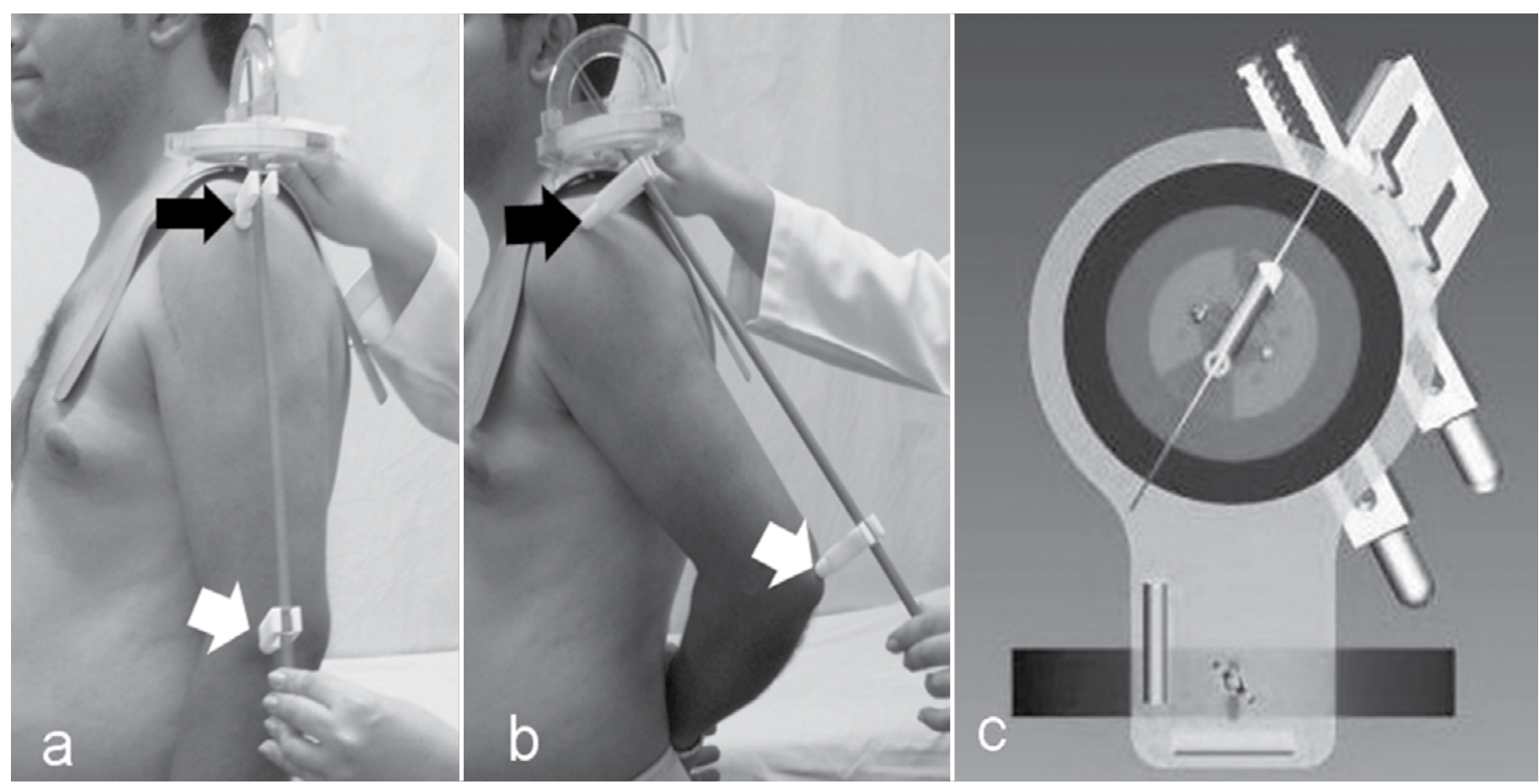

Figure 2 - Positioning of the bi-goniometer on the shoulder, with patient standing upright. a) Anatomical position, with reference points on the lateral edge of the acromion (black arrow) and on the lateral epicondyle (white arrow). b) Medial rotation position of the shoulder, with reference points on the lateral edge of the acromion (black arrow) and at the tip of the olecranon (white arrow). c) Axis of the angle y, viewed from above, corresponding to extension and adduction of the shoulder (Department of Mechanical Engineering, Mackenzie Presbyterian University). 


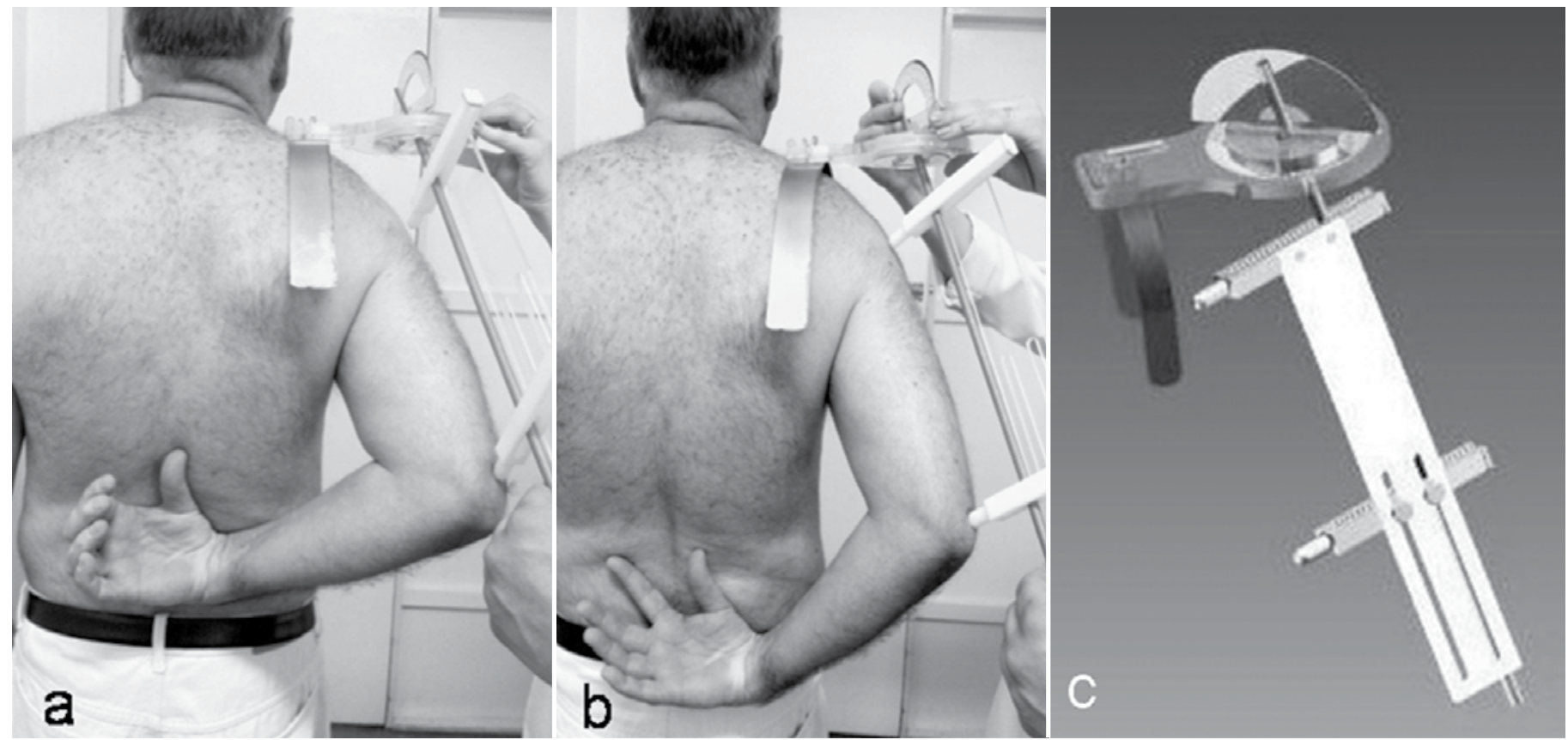

Figure 3 - Posterior view of the positioning of the bi-goniometer for measuring the medial rotation of the affected shoulder. a) Maximum vertebral level reached (T10). b) Corrected vertebral level (L1). c) Axis of the angle $\mathrm{x}$ in the frontal plane, corresponding to abduction and medial rotation of the shoulder (Department of Mechanical Engineering, Mackenzie Presbyterian University).

angular variation of the $y$ axis (angle y on affected side minus y on unaffected side, in medial rotation).

The mean, standard deviation (SD) and confidence interval $(\mathrm{CI})$ were calculated for all the variables analyzed. Regarding the angle $\mathrm{x}$ on the unaffected and affected sides, in the anatomical position, the mean difference in comparing the 70 patients was within the CI, with $\mathrm{p}>0.005$, and thus the hypothesis of equality between the measurements was accepted $(p=0.681)$ (Figure 4). The same was found in calculating the difference for the angle y on the unaffected and affected sides, in the anatomical position, with $p=0.545$ (Figure 5).

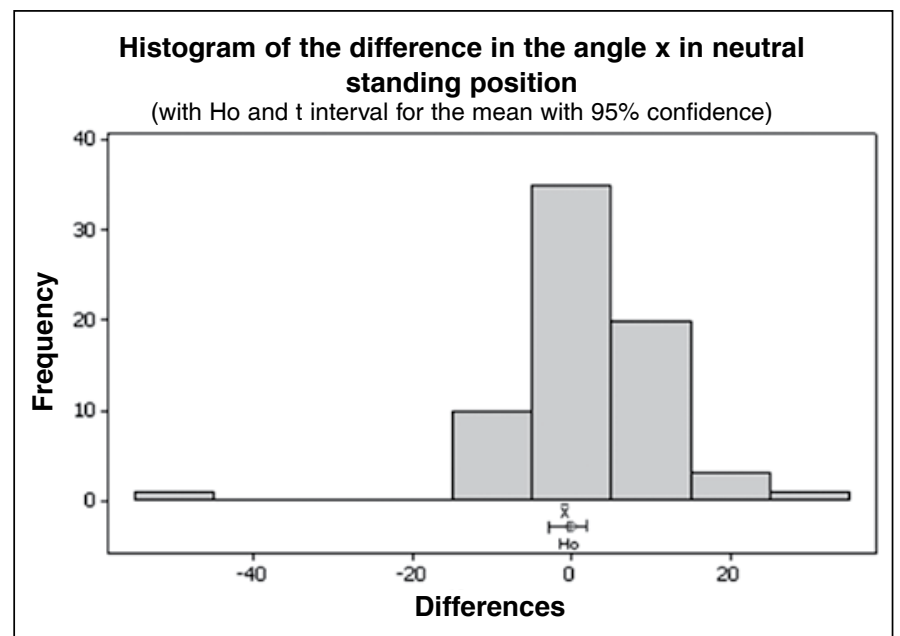

Figure 4 - Distribution of the difference in $\mathrm{x}$ in the anatomical upright standing position between the affected and unaffected sides.

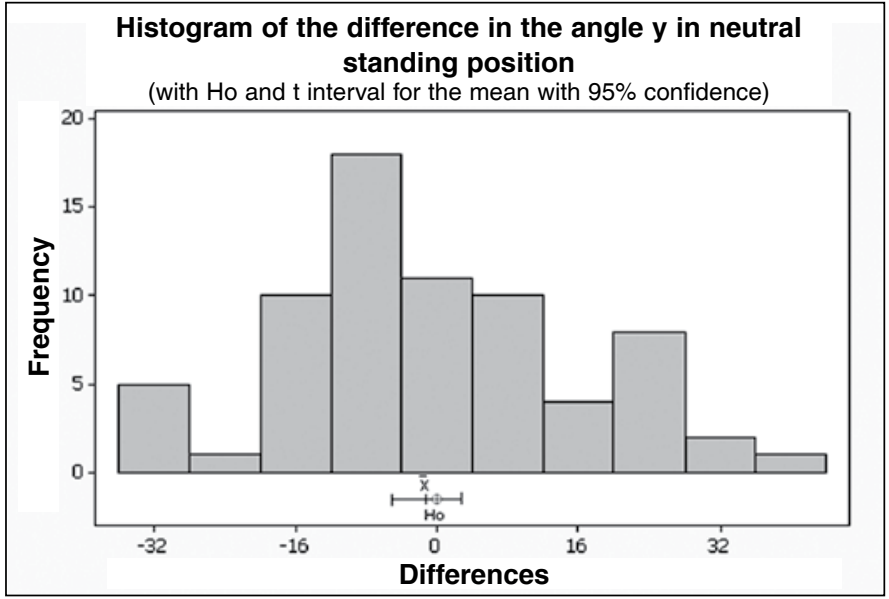

Figure 5 - Distribution of the difference in y in the anatomical upright standing position between the affected and unaffected sides.

\section{RESULTS}

In evaluating the variable of the angle $\mathrm{x}$ for the 70 patients, comparing this angle between the unaffected and affected sides in medial rotation, the mean difference was within the $\mathrm{CI}$, with $\mathrm{p}=0.002$. It was therefore concluded that the measurements were not equal for the unaffected and affected sides, such that they were greater on the affected side (Figure 6).

In relation to the variable of the angle $y$ for the 70 patients, comparing this angle between the unaffected and affected sides in medial rotation, the mean difference was within the $\mathrm{CI}$, with $\mathrm{p}=0.000$. It was therefore concluded that the measurements were not equal for the unaffected and affected sides, such that 
they were greater on the affected side (Figure 7).

The maximum vertebral level reached in medial rotation with the thumb outstretched on the unaffected side ranged from $\mathrm{T} 3$ to $\mathrm{T} 12$, and $46 \%$ of the cases were between $\mathrm{T} 8$ and T9. On the affected side, the maximum vertebral level reached with the thumb outstretched ranged from T6 to the trochanter. When the affected limb was repositioned in medial rotation in accordance with the angular values of the normal side, the levels ranged from $\mathrm{T} 6$ to the trochanter, but with a different percentage distribution, such that $57.13 \%$ of the patients reached levels between the sacrum, gluteus and trochanter (Table 1).

The variable of maximum elbow flexion on the unaffected and affected sides was analyzed, comparing these measurements for the 70 patients. It was concluded that the maximum elbow flexion on the unaffected and affected sides was not equal, such that it was greater on the unaffected side $(p=0.000)$ (Figure 8). In the same way, in comparing the patients who reached vertebral levels higher than S1 with their

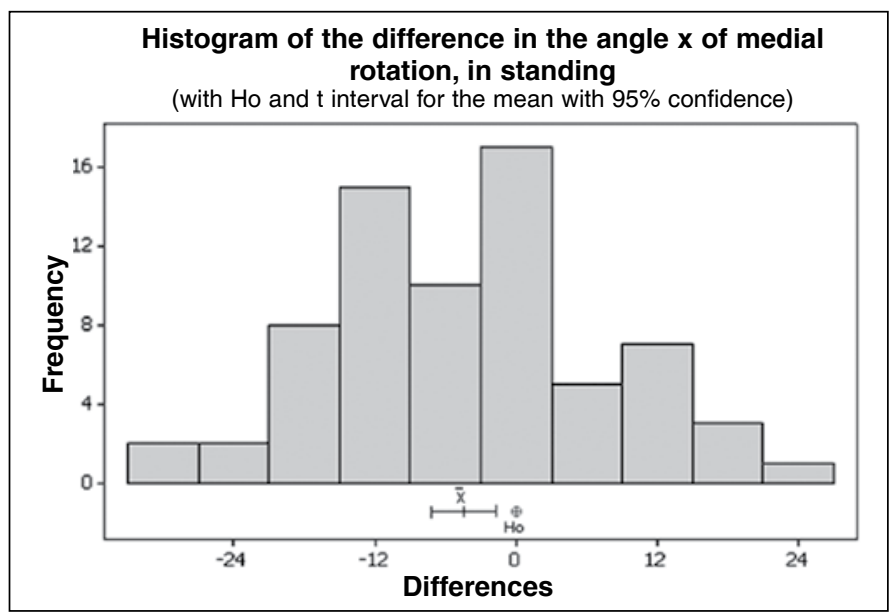

Figura 6 - Distribuição da diferença entre a rotação medial x para o lado acometido e o não acometido.

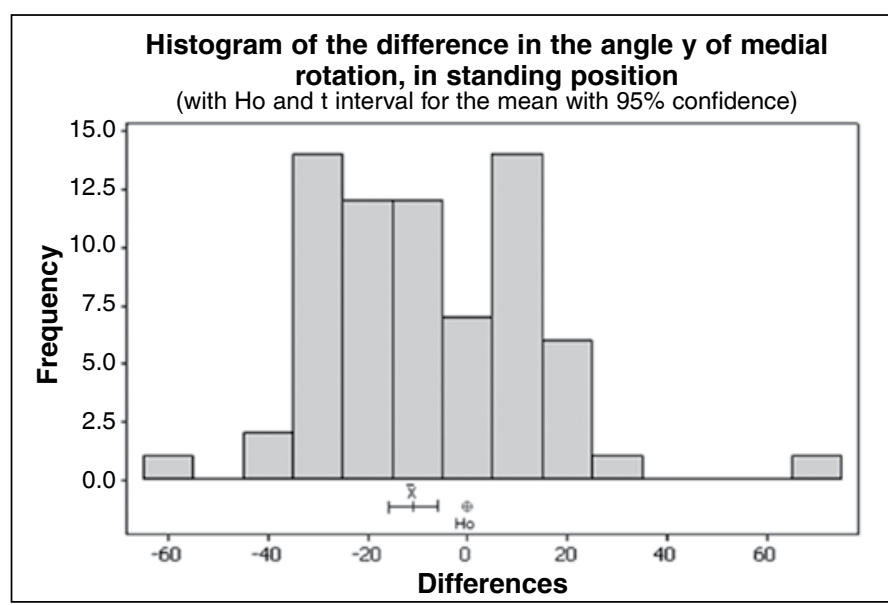

Figura 7 - Distribuição da diferença entre a rotação medial y para o lado acometido e o não acometido.
Table 1 - Incidence of vertebral levels for the unaffected, affected and corrected affected sides.

\begin{tabular}{c|c|c|c}
\hline $\begin{array}{c}\text { Vertebral } \\
\text { level }\end{array}$ & $\begin{array}{c}\text { Unaffected } \\
\text { side (\%) }\end{array}$ & Affected side (\%) & $\begin{array}{c}\text { Corrected } \\
\text { affected side (\%) }\end{array}$ \\
\hline T3 & 1.43 & 0 & 0 \\
\hline T4 & 2.86 & 0 & 0 \\
\hline T5 & 2.86 & 0 & 0 \\
\hline T6 & 5.71 & 1.43 & 1.43 \\
\hline T7 & 17.14 & 1.43 & 0 \\
\hline T8 & 24.29 & 4.29 & 1.43 \\
\hline T9 & 21.42 & 7.14 & 2.86 \\
\hline T10 & 14.29 & 7.14 & 7.14 \\
\hline T11 & 2.86 & 14.29 & 1.43 \\
\hline T12 & 7.14 & 8.57 & 5.71 \\
\hline L1 & 0 & 4.29 & 2.86 \\
\hline L2 & 0 & 10 & 8.57 \\
\hline L3 & 0 & 5.7 & 4.29 \\
\hline L4 & 0 & 0 & 5.72 \\
\hline L5 & 0 & 12.86 & 1.43 \\
\hline Sacrum & 0 & 8.57 & 15.71 \\
\hline Gluteus & 0 & 12.86 & 25.71 \\
\hline Trochanter & 0 & 1.43 & 15.71 \\
\hline Total & 100 & 100 & 100 \\
\hline
\end{tabular}

Legend: $T$ - thoracic; $L$ - lumbar.

Source: medical files at Irmandade Santa Casa de Misericórdia de São Paulo.

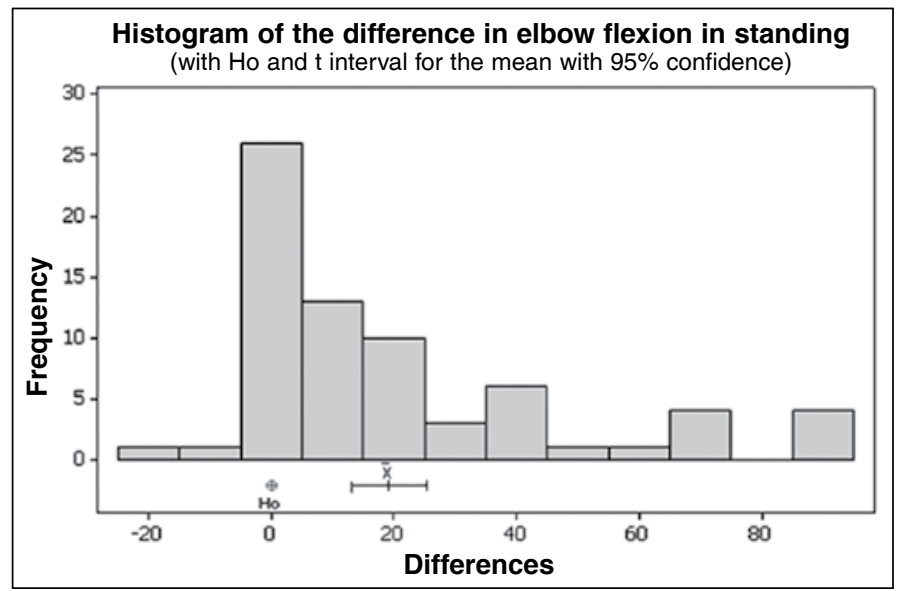

Figure 8 - Distribution of the difference in elbow flexion in standing position between the affected and unaffected sides.

affected limb, with unaffected individuals, the elbow flexion was also greater on the unaffected side.

In evaluating the variables of difference in maximum vertebral level reached and $\Delta \mathrm{x}$ variation (angle $\mathrm{x}$ on the affected side minus $x$ on the unaffected side, in medial rotation), it was concluded that there was a decreasing linear relationship between these variables, i.e. the greater the angle of the diaphysis axis is, the smaller the variation in the vertebral level achieved is $(p=0.044)$ (Figure 9).

Following this, the linear correlation was calculated between the variables of difference in the maximum vertebral level reached and $\Delta y$ variation (angle y on the 
affected side minus $y$ on the unaffected side, in medial rotation), and the value obtained was -0.231 . At the significance level of $5 \%$, this value was zero $(\mathrm{P}=0.054)$, and it was concluded that there was no well-established linear relationship between these variables (Figure 10).

Complaints of shoulder pain did not influence the vertebral level reached with the affected limb $(p=0.309)$. However, through correlating the elbow flexion on the side with medial rotation and the presence of pain, it was concluded that patients with pain performed greater compensatory flexion of the elbow $(p=0.004)$.

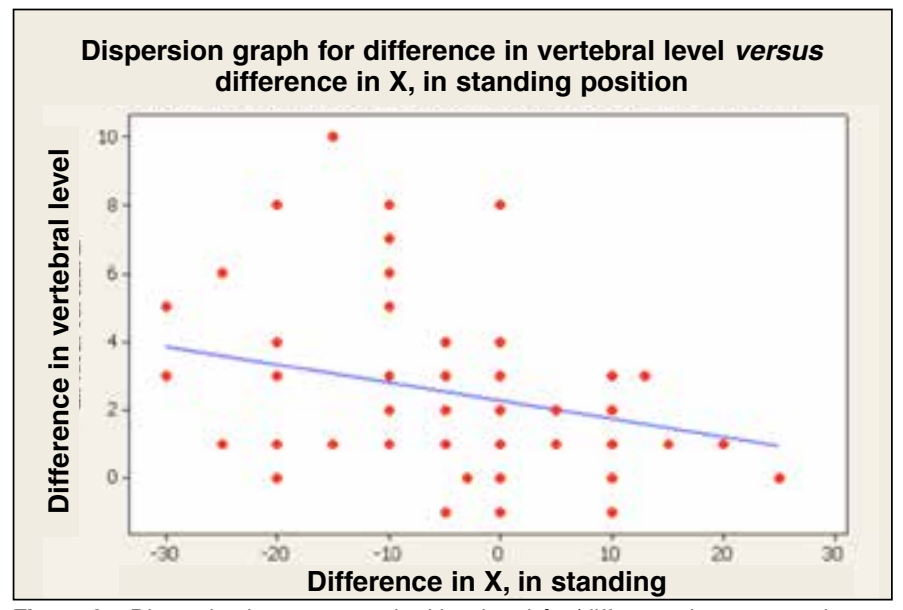

Figure 9 - Dispersion between vertebral level and $\Delta x$ (difference between angle $\mathrm{x}$ on the affected side and $x$ on the unaffected side, in medial rotation).

Dispersion graph for difference in vertebral level versus difference in $\mathrm{Y}$, in standing position

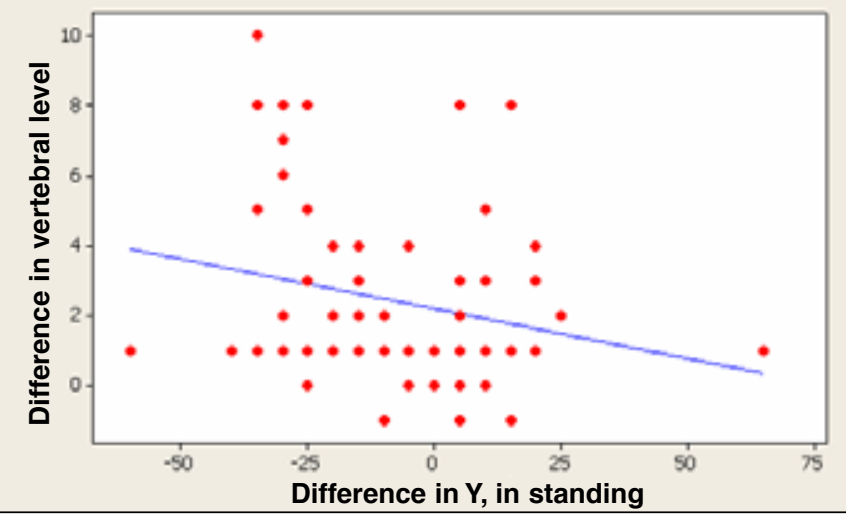

Figure 10 - Dispersion between vertebral level and $\Delta y$ (difference between angle $y$ on the affected side and $y$ on the unaffected side, in medial rotation).

\section{DISCUSSION}

Measurement of medial rotation of the shoulder is very complex, and there is no standard for arm positioning, thus making such measurements imprecise ${ }^{(13)}$. In our study, we sought to standardize the reference points of the axis of the humeral diaphysis, so as to make measurements on medial rotation of the shoulder using the visual scale method less subjective. We also maintained the same measurement routine: while examiner E1 always positioned the instrument, E2 always made the measurements.

Although Armstrong et $a l^{(7)}$ found that measurements of medial rotation of the shoulder by means of visual estimation presented moderate reproducibility, Andrade et $a l^{(1)}$ showed through comparing this method with goniometry that measurements using a visual scale were more reproducible. In our study, in testing the measurements of medial rotation according to the maximum vertebral level reached, we obtained high concordance between the two examiners, thus confirming from the statistical analysis that the measurements were reproducible.

In accordance with the findings of Mallon et $a l^{(4)}$, who defined that hand positioning behind the back takes place in three distinct movements, we developed a numerical form of measurement of medial rotation of the shoulder that took into consideration the initial movement of abduction and medial rotation, measured using the angle of the $\mathrm{x}$ axis. This movement takes place mainly in the shoulder joint. Subsequently, there are extension, adduction and medial rotation movements, performed by the scapulothoracic joint, which we measured using the angle of the y axis. Lastly, we measured the angle of elbow flexion using conventional goniometry: this joint is the main agent responsible for medial rotation from the midline of the sacrum, and it may move the hand 17 to $20 \mathrm{~cm}$ proximally, corresponding to a gain of seven to eight levels in normal individuals ${ }^{(14)}$.

In our study, we concluded that the maximum elbow flexion on the affected side was shown to be statistically less than on the unaffected side, for all the vertebral levels reached, and that patients who only reached levels lower than the lumbar level L5 had lower degrees of elbow flexion. This may be explained by the studies of Mallon et $a l^{(4)}$, who demonstrated that elbow flexion was the main agent responsible for reaching vertebral levels between S1 and T10.

In evaluating patients with complaints of pain in the affected shoulder, this variable was not correlated with lower vertebral levels. However, these patients' elbow flexion was shown to be greater $(p=0.004)$, which may have been in an attempt to compensate for the medial rotation deficit. It should be noted that none of these individuals presented any pain or limitation of elbow movements.

Although Andrade et al ${ }^{(1)}$ did not find any correlation between the angular measurement of medial rotation of the shoulder at $90^{\circ}$ of abduction with the patient in a supine position and the vertebral level observed on the visual scale, given that these are different methods that cannot 
be compared, we were able to establish a decreasing linear relationship between the variation in vertebral level on the visual scale and the variation in angular values obtained using bi-goniometry in the $\mathrm{x}$ axis. Thus, the greater the compensatory angle of the affected upper limb was, in relation to the normal side $(\Delta \mathrm{x})$, the greater the variation in the corrected vertebral level was $(p=0.044)$. On the other hand, in comparing $\Delta y$ with the variation in vertebral level, the statistical value at the significance level of $5 \%$ was very close to $0.05(\mathrm{p}=0.054)$, which suggests that we cannot affirm that there is a well-defined relationship. However, a strong association was shown between the variables, and thus the error may have been in the sample or in the measurement.

Our work clearly demonstrated that the value of the vertebral level measured in medical practice is overestimated, given that with repositioning of the affected upper limb according to the angular values found in the unaffected upper limb, there was a decrease in these measurements by at least one level, for all the patients studied.

Greene and Heckman ${ }^{(11)}$ cited the maximum vertebral level reached as a measurement of interest to shoulder surgeons because of its functional importance in activities of daily living, such as hygiene, closure of bras by women and removal of wallets from back pockets of trousers/pants. Thus, although the value of medial rotation of the shoulder may be overestimated in the measurements, it is important to bear in mind that this measurement is a form of compensation of arm positioning for functional adaptation, thus making upper limbs with movement limitations functionally as close as possible to the normal side.

In conformity with the literature, in which the maximum vertebral level reached is cited as ranging from T6 to T10 in normal individuals ${ }^{(11)}, 82.86 \%$ (58

\section{REFERENCES}

1. Andrade JA, Leite VM, Teixeira-Salmela LF, Araújo PMP, Juliano Y. Estudo comparativo entre os métodos de estimativa visual e goniometria para avaliação das amplitudes de movimento da articulação do ombro. Acta Fisiátrica. 2003;10(1):12-6

2. American Academy of Orthopaedic Surgeons. Joint motion: method of measurement and recording. Edinburgh: British Orthopaedic ASSn; 1965.

3. Kumar V, Satku S. Documenting rotation the glenohumeral joint. A technical note. Acta Orthop Scand. 1994;65:483-4.

4. Mallon WJ, Herring CL, Sallay PI, Moorman CT, Crim JR. Use of vertebral levels to measure presumed internal rotation at the shoulder: A radiographic analysis. J Shoulder Elbow Surg Am. 1996;5(4):299-306.

5. Edwards T, Bostick R, Greene C, Baratta R, Drez D. Interobserver and intraobserver reliability of the measurement of the shoulder internal rotation by vertebral level. J Shoulder Elbow Surg Am. 2002;11(1):40-2.

6. Ginn KA, Cohen ML, Herbert RD. Does hand-behind-back range of motion accurately reflect shoulder internal rotation? J Shoulder Elbow Surg Am. 2006;15(3):311-4.

7. Armstrong AD, MacDermid JC, Chinchalkar S, Stevens RS, King GJW. Reability of range-of-motion measurement in the elbow and forearm. J Shoulder Elbow Surg Am. 1998;7(6):573-80. cases) of our sample were within this range on the unaffected side. On the other hand, on the affected side, only $21.43 \%$ ( 15 cases) of the measurements were within this interval and, after correction of the angular positioning of the humeral diaphysis, this number went down to $12.86 \%$ (nine cases).

Taking into account that hand reach lower than vertebral level L5 is functionally very poor for performing activities of daily living, we can conclude that compensation for the trunk is very important, given that 16 patients $(22.86 \%)$ actively reached the sacrum, gluteus and trochanter levels with the upper limb, and after the correction, the number of patients who were unable to reach levels above the sacrum increased to 40 patients $(57.13 \%)$.

Although Andrade et a $l^{(1)}$ suggested that a new device should be created for measuring medial rotation of the shoulder, given the difficulty in determining the center of rotation of the movements of this joint, we did not find any study developing such equipment in the literature. Our study was a pioneer in attempting to develop a new measuring instrument and, although we tested its applicability, we suggest that it should be reevaluated in new studies in order to confirm its reliability.

Measurement of the medial rotation of the shoulder using a bi-goniometer is relatively fast and easy. However, because this instrument is not available on the market, there is some difficulty in conducting comparative studies, which makes its applicability limited, from a practical point of view.

\section{CONCLUSION}

In conclusion, we can affirm that measurements of medial rotation according to vertebral levels do not correspond to the real values, since they vary according to the positioning of the humeral diaphysis.

8. Herrington L. Glenohumeral joint: internal e external rotation range of motion in javelin throwers. Br J Sports Med. 1998;32(3):226-8.

9. Green S, Buchbinder R, Forbes A, Bellamy N. A standardized protocol for measurement of range of movement of the shoulder using the Plurimeter-V inclinometer and assessment of its intrarater and interrater reliability.Arthritis Care Res. 1998;1(1):43-52.

10. Richards R, Bigliani L, Gartsman G, lannotti J, Zuckerman J. A standardized method for the assessment of shoulder function. J Shoulder Elbow Surg Am. 1994;3:347-52.

11. Greene WB, Heckman JD. The shoulder. In: The clinical measurement of joint motion. Rosemont: Americam Academy Orthopaedic Surgeons; 1994. p. $15-26$.

12. Wakabayashi I, Itoi E, Minagawa $H$, Kobayashi M, Seki N, Shimada $Y$, et al. Does reaching the back reflect the actual internal rotation of the shoulder? J Shoulder Elbow Surg Am. 2006;15(3):306-10.

13. Matsen FA. Presidential address: American shoulder and elbow surgeons, Tenth Anniversary, Annual Meeting. J Shoulder Elbow Surg.1992;1:63-4.

14. Hollinshead WH. Anatomy for surgeons. Back and Limbs. 3nd ed. Philadelphia: Harper and Ro; 1982. 